\title{
La hibridación de los modelos pedagógicos de aprendizaje cooperativo y educación aventura como estrategia didáctica para la mejora de la convivencia y la gestión de conflictos en el aula: una experiencia práctica desde las clases de educación física y tutoría

\author{
The hybridization of the pedagogical models of cooperative learning and \\ conflict management in class: a practical experience from the physical \\ education and tutoring lessons
} adventure education as a didactic strategy for enhancing the coexistence and
}

Rafael Merino, Jorge Lizandra

Universitat de València (España)

Resumen. El presente estudio adopta un enfoque cualitativo para profundizar en la mejora de la convivencia y la gestión de conflictos desde el área de Educación Física y la tutoría. A partir de una evaluación diagnóstica, se diseñó una Unidad Didáctica innovadora en la que se hibridaron los modelos pedagógicos de educación aventura y aprendizaje cooperativo, introduciendo el contenido de educación emocional en una clase de $1^{\circ}$ de Educación Secundaria Obligatoria. De forma paralela, se realizó un proceso de investigación basada en un estudio de caso en el que se recogieron las percepciones y valoraciones de la experiencia de innovación del alumnado, del docente especialista de la asignatura y se triangularon con el registro anecdótico observacional del investigador. Los resultados obtenidos evidencian una contribución positiva en la formación de un alumnado que mostraba carencias en la convivencia y la resolución de conflictos, observándose mejoras en su educación emocional. Además, la hibridación de modelos pedagógicos se ha mostrado como una buena contribución a la didáctica de la Educación Física, en la medida en la que permite la integración de contenidos transversales y propios de la asignatura de Educación Física.

Palabras clave: convivencia, resolución de conflictos, hibridación, modelos pedagógicos, didáctica, educación secundaria, adolescencia.

\begin{abstract}
The present study adopts a qualitative approach to deepen the enhance of coexistence and conflict management from the area of Physical Education and tutoring. Based on a diagnostic evaluation, an innovative Didactic Unit was designed in which the pedagogical models of Adventure Education and Cooperative Learning were hybridized, introducing the content of social and emotional learning in a 1st Obligatory Secondary Education curse. In parallel, a research process based on as a case study was carried out in which the perceptions and appreciations of the innovation experience of the students, the specialist teacher of the subject were collected and triangulated with the observational anecdotal record of the researcher. The results show a positive contribution in the behavioural training of a student body that showed deficiencies in coexistence and conflict resolution, observing improvements in their emotional education. In addition, the hybridization of pedagogical models has been shown as a good contribution to the didactics of Physical Education, insofar as it allows the integration of both, cross-sectional and own contents of the Physical Education subject.
\end{abstract}

Keywords: coexistence, conflict resolution, hybridization, pedagogical models, didactics, secondary education, adolescence.

\section{Introducción}

La mejora de la práctica educativa es una de las principales preocupaciones del profesorado en general y, por tanto, también en el ámbito de la Educación Física (EF). Los constantes cambios sociales generan nuevas exigencias educativas que precisan de enfoques alternativos a los estilos de enseñanza tradicional. En ese sentido, los modelos pedagógicos han emergido como un intere-

Fecha recepción: 26-11-20. Fecha de aceptación: 29-09-21 jorge.lizandra@uv.es Jorge Lizandra sante marco de referencia en la didáctica de la EF (Peiró \& Julián, 2015). Metzler (2017), los define como planteamientos metodológicos de carácter global y multifactorial capaces de integrar teorías de la enseñanza y el aprendizaje y elementos curriculares a la vez que se tienen en cuenta cuestiones didácticas como la gestión del aula, el control del proceso y la evaluación del aprendizaje del alumnado. Este enfoque integrado permite al mismo tiempo que se imparten contenidos disciplinarios, incidir sobre cuestiones nucleares de la educación como la resolución de conflictos y la convivencia en las aulas. 
García-Hierro y Cubo (2009) definen la convivencia escolar como la interrelación entre los diferentes miembros de un proceso educativo, que incide significativamente en el desarrollo tanto ético, socioafectivo como intelectual del alumnado. En ese sentido, se puede inferir que la escuela es uno de los principales agentes socializadores durante la infancia y la adolescencia (Abramovay, 2005). Es por ello que preocupa el incremento de comportamientos agresivos y violentos que han proliferado en las escuelas (Palacio \& BermejoGarcía, 2021), por lo que las instituciones educativas reconocen la necesidad de ofrecer herramientas al alumnado que mejoren sus relaciones con las personas y el entorno.

La asignatura de EF, por el hecho de realizarse en un espacio diferente al aula y dadas las mayores posibilidades de interacción entre iguales que ofrece el movimiento, la convierte en un contexto propicio para la generación de conflictos (Martín \& Ríos, 2014). Una de las revisiones sistemáticas realizadas por Jiménez-Barbero, Jiménez-Loaisa, González-Cutre, Beltrán-Carrillo, Llor-Zaragoza \& Ruiz-Hernández (2020) revelan la importancia de esta área y los programas de EF pudiendo llegar a mejorar las habilidades del alumnado para hacer frente a la violencia y el acoso escolar. Del mismo modo, conocidas las características contextuales de la asignatura y las situaciones que pueden ocasionarse, las clases de EF son igualmente escenarios especialmente propicios para progresar en la mejora de la convivencia y en la resolución y mediación de conflictos (Fraile-Aranda, López-Pastor, Ruíz-Omeñaca \& VelázquezCallado, 2008). Tanto es así que además de ser una asignatura bien valorada por el alumnado, (González, Gómez-López \& Granero-Gallegos, 2019), las relaciones que en ella se establecen, pueden mediar como facilitadores e inhibidores de la práctica de AF (Lizandra \& Peiró-Velert, 2020). No obstante, diversos autores recomiendan que las acciones educativas sean coordinadas desde diferentes ámbitos de actuación, bien sea a nivel curricular, o desde el plan de acción tutorial (Funes, 2000; Moreno, 2010). En ese sentido, Monzonís (2015) encontró ventajas en la introducción de un plan de acción coordinado entre la asignatura de EF y la tutoría, especialmente para la atención a la diversidad del alumnado.

Distintos estudios han mostrado los modelos pedagógicos como una metodología apropiada tanto para la mediación como para el trabajo interdisciplinar. Concretamente, de entre la variedad de modelos pedagógicos identificados y descritos hasta el momento en la literatura (Fernández-Rio, Calderon, Hortiguela, PerezPueyo \& Aznar-Cebamanos, 2016), los modelos de aprendizaje cooperativo (AC) y de Educación Aventura (EA), son dos de los que mejor han contribuido a la mejora de la convivencia en las aulas (Baena-Extremera, 2011; Caballero, Sayazo, Dominguez, Perez \& Parra, 2016; Fernández-Río \& Méndez-Giménez, 2016). Asimismo, se han encontrado revisiones sistemáticas sobre la hibridación de modelos pedagógicos como la de González-Víllora, Evangelio, Sierra-Días \& FernándezRío (2019) en las que se manifiesta una ventaja de implementación de los modelos pedagógicos híbridos frente a la aislada.

Según Johnson y Johnson (1999), el modelo de AC es aquel en el que se realiza un trabajo en grupos, y el alumnado trabaja de forma conjunta para conseguir unas metas comunes fijadas previamente. Estos autores identifican cinco características indispensables en el desarrollo del AC en la práctica educativa:

Interdependencia positiva: el equipo precisa de todos y cada uno de sus integrantes para resolver la tarea.

Interacción promotora: los miembros del grupo favorecen y estimulan los aprendizajes del resto de los compañeros para alcanzar los objetivos grupales.

Responsabilidad Individual: cada uno de los integrantes desde su rol suma con su contribución a la consecución de los objetivos comunes.

Habilidades interpersonales y de trabajo en pequeño grupo: desarrollo eficaz de habilidades sociales como la empatía y la asertividad para aceptar y apoyar las opiniones del resto del grupo, así como de ser capaz de gestionar positivamente los conflictos interpersonales.

Autoevaluación grupal: los miembros del grupo reflexionan sobre la actividad realizada para determinar las acciones eficaces y tomar decisiones sobre aquellas que no lo fueron.

Por otro lado, el modelo de EA, según BaenaExtremera (2011) se define como el conjunto de actividades variadas que interaccionan con el entorno natural, conjugando elementos como el peligro real o aparente, en el que los resultados son inciertos en tanto que pueden ser influidos tanto por los participantes como por las circunstancias del entorno. En ese sentido, Navarro, Collado y Pellicer (2020) identifican igualmente las siguientes cinco características:

- Resolución de problemas: el alumnado se ve enfrentado a un problema que debe solucionar en un medio cambiante, que previamente debe conocer y adap- 
tarse.

Superación de barreras: aunque habitualmente se hace referencia a barreras de tipo físico, también entran en juego las de tipo mental y personales, como el miedo o la capacidad de resiliencia.

Cooperación: muchos de los problemas se resuelven con mayor eficacia a través de la cooperación con los compañeros.

- Uso creativo de espacios y materiales: especialmente importante cuando las actividades son simuladas en los centros educativos, pues requiere de la capacidad de aprovechar los recursos disponibles para crear espacios de aventura.

- Contexto lúdico: es imprescindible que todas las actividades tengan lugar en un ambiente festivo, de juego, para motivar a los estudiantes y disminuir la presión derivada del riesgo existente.

Vistas las características de ambos modelos pedagógicos cabe indicar que autores como Velázquez (2012) aplicaron el modelo de AC obteniendo buenos resultados en la mejora de las relaciones sociales y la adquisición de competencias sociales. Asimismo, Sáez de Ocáriz, et al. (2014) concluyeron que el trabajo cooperativo puede optimizar el desarrollo de habilidades y competencias sociales que mejoren la convivencia escolar. Con respecto al modelo pedagógicos de EA, BaenaExtremera (2011) afirma que su desarrollo en el medio natural, favorece la participación activa y despierta un gran interés entre el profesorado y el alumnado, aun siendo igualmente aplicables en el aula. Hortiguela, Perez-Pueyo y Fernández-Rio (2020), destacan la necesidad de aplicar este tipo de modelos pedagógicos especialmente en contextos desfavorecidos, en los que las actitudes suelen ser menos adecuadas. De manera más categórica, Aubert, Bizkarra y Calvo (2014) afirman que la EF es considerada un elemento socializador que propicia la cohesión social. Por consiguiente, este tipo de estudios validan la idea de Sánchez-Alcaraz (2014) de entender la EF como una disciplina motivante para el alumnado, promotora de relaciones interpersonales y comunicación que, bien trabajadas, puede desarrollar cualidades sociales y personales como la autoestima, la solidaridad o la cooperación.

En los últimos años, autores como el propio Fernández-Río (2015) o Pérez-Pueyo et al. (2017) han introducido la posibilidad de diseñar hibridaciones de modelos pedagógicos que permiten complementar y ampliar los beneficios obtenidos por el desarrollo de propuestas metodológicas de un modelo único. Se encuentran algunos precedentes en trabajos recientes como el de Guijarro, Evangelio, González-Víllora y AriasPalencia, (2020), con la hibridación del modelo comprensivo de iniciación deportiva con el aprendizaje cooperativo, o el de Valero-Valenzuela, Gregorio Garcia, Camerino y Manzano (2020), que desarrollaron una hibridación del modelo de responsabilidad personal y social y la EF gamificada. Igualmente, existe un precedente reciente de hibridación de los modelos de AC y EA con entornos gamificados para la carrera de orientación en el que trabajo de Lamoneda-Prieto, González-Víllora y Fernández-Río (2020), destacando la adquisición de aprendizajes y competencias de un mayor nivel educativo que los obtenidos con métodos analógicos de carrera de orientación deportiva.

Con todo ello, se podría concluir que la enseñanza de la EF basada en modelos pedagógicos ha supuesto un cambio en el enfoque didáctico centrado en las necesidades del alumnado. Además, las interacciones y coincidencias entre las características de varios modelos, junto con la necesidad de establecer estrategias didácticas para hacer frente a las limitaciones en el contexto, ha llevado a diferentes profesionales a proponer la hibridación de modelos pedagógicos. No obstante, su reciente incorporación en el marco teórico, práctico y epistemológico de la EF, precisa del planteamiento y estudio en profundidad de nuevas experiencias que permitan seguir indagando y conociendo los beneficios de la enseñanza basada en modelos pedagógicos. En ese sentido, el siguiente trabajo tiene dos objetivos. El primero es describir la experiencia de diseño, desarrollo y evaluación de una Unidad Didáctica (UD) en la que se hibridaron los modelos pedagógicos de EA y AC como estrategia metodológica para mejorar la convivencia y la gestión de conflictos desde las asignaturas de EF y tutoría. El segundo es conocer las percepciones, valoraciones y el grado de satisfacción del alumnado y profesorado tras haber vivenciado la UD diseñada.

\section{Metodología}

\section{Muestra}

El presente estudio se realizó en un centro público de educación secundaria de la provincia de Valencia durante el desarrollo del periodo de prácticas del Máster en Profesor/a de Educación Secundaria en el curso 2019/ 2020. Concretamente, la experiencia se desarrolló en un grupo de $1^{\circ} \mathrm{ESO}$ caracterizado por ser el que mayores problemas de convivencia computaba. El grupo estaba constituido por 24 estudiantes, 13 chicas y 11 chicos, con edades comprendidas entre los 12 y los 13 años. 
Destaca la predisposición del tutor del grupo que no solo accedió a que se realizara la experiencia, sino que la incorporó en su programación de la asignatura y además permitió trasladar fases de la investigación al espacio de tutoría, esencial en el seguimiento de la experiencia.

\section{Procedimiento y consideraciones éticas}

El trabajo de campo fue desarrollado durante el segundo y tercer trimestre del curso 2019-2020, por el estudiante en prácticas después de haber realizado una consulta bibliográfica extensa sobre el marco teórico relacionado con los modelos pedagógicos en el área de EF, así como diferentes aplicaciones e hibridaciones recientemente publicadas. Este trabajo previo, junto con el apoyo y seguimiento del tutor de la universidad, realizando sucesivas reuniones y explicaciones sobre los elementos clave del marco teórico y su implementación, permitieron adquirir el aprendizaje y la experiencia para desarrollar el presente estudio. Una vez determinados los ejes sobre los que se articularía la investigación de la propuesta innovadora y de haber pedido permiso al especialista del área, se configuró la UD.

Tras recibir el consentimiento del centro para realizar la intervención, se exploró y se configuraron las herramientas más oportunas para la obtención de datos, asegurando la confidencialidad y protegiendo en todo momento la identidad de todos los y las participantes en el trabajo.

\section{Descripción de las fases de intervención}

Siguiendo a Caballero et al. (2006), el diseño y desarrollo de la intervención se articuló en las tres fases; fase preactiva, activa y postactiva (ver tabla 1 ).

La fase preactiva ( $2^{a}$ quincena enero 2020) consistió en la observación y conocimiento del alumnado. El objetivo general fue conocer las características del grupo y el tipo de relaciones que entre ellos se daban, de manera que la planificación y programación de la intervención diseñada pudiera suponer un aprendizaje más significativo. Fue en este momento cuando se identificaron diferentes problemas de convivencia y comportamiento en el grupo sobre los que se consideró necesario intervenir. Tras comentarlo con el docente titular y el tutor de las prácticas, se decidió profundizar en el conocimiento del grupo y orientar la intervención en la fase activa hacia el trabajo cooperativo, como alternativa al enfrentamiento o competición que agravaba los problemas de conducta.

La fase activa (febrero 2020) consistió en el diseño y puesta en práctica de la intervención en un total de 12 sesiones (figura 1). Conocer el marco teórico y práctico de los modelos pedagógicos y la posibilidad de plantear su hibridación resultó esencial para optar por el diseño de la UD. Se entendió que además de realizar un trabajo cooperativo, hacerlo desde un planteamiento de deporte aventura, supondría exponer al alumnado ante un contexto con el que no estaban familiarizados. De esta manera se lograría, por una parte, captar su interés y por otra, equilibrar los niveles de ejecución. Todo ello encaminado a reducir el carácter competitivo atribuido de manera generalizada a la clase de EF y a crear un contexto proclive a la colaboración. Así pues, conociendo que la cooperación es una de las características clave de los modelos de aventura de Baena-Extremera (2011), se consideró interesante optar por la hibridación de los modelos de EA y AC. Para ello, tras revisar distintos modelos se decidió, tomar las fases delAC de FernándezRio (2003) y la progresión de actividades de la EA de Parra, Caballero y Domínguez (2009), para diseñar la hibridación, pues se consideró que permitían integrar las diferentes fases de cada uno de los modelos, con las actividades propuestas y ajustarlas a la distribución temporal prevista. Concretamente, la UD se programó desde los bloques de contenido 2, 3 y 5 del área de EF del Real Decreto 1105/2014, de 26 de diciembre. La hibridación de modelos pedagógicos se planteó, desde el bloque 2 (juegos y deportes) mediante el goalball o desafíos manipulativos cooperativos. También, el bloque 3 (Actividades adaptadas al medio) mediante gymkhanas cooperativas de orientación y búsqueda en el entorno próximo. Mientras que en el bloque 5 (Elementos transversales a la asignatura), se introdujo la educación emocional en el aula desde las cuatro sesiones de tutoría. Cabe indicar que se trató de ofrecer un rol protagonista al alumnado que, aunque no disponía de experiencia previa en el trabajo cooperativo ni en contextos de aventura, sí pudo recibir la información y orientaciones necesarias para participar activamente en las distintas sesiones.

La fase postactiva (marzo-mayo 2020) sirvió para establecer los espacios para el análisis de los resultados y la reflexión de lo acontecido en la intervención. Por tanto, fue una fase esencial en el proceso de investigación de la innovación educativa basada en la hibridación de modelos pedagógicos. Para ello, además del registro que como docente en prácticas se llevaba de toda la intervención, se consideró necesario contar con la valoración tanto del alumnado como del docente titular. De esta manera, se trató de ser coherente con la intención 
de reconocer al alumnado como protagonista e interlocutor válido de la experiencia, a la vez que se conocía la opinión del docente y la intención de integrar la propuesta en su programación.

\section{Instrumentos para la recogida de datos}

Durante el desarrollo de las fases de la intervención se han ido tomando datos que han tenido diferentes objetivos y finalidades, contribuyendo de una u otra manera a la investigación de la propuesta de innovación.

En la fase preactiva, una vez observados los problemas de conducta y convivencia, se decidió utilizar el cuestionario validado en Monzonís (2015) dirigido a reconocer la calidad de la competencia social del alumnado, que fue administrado en la clase de tutoría. Además, el ránking sociométrico del propio Monzonís, permitió identificar los/las líderes del grupo y las personas con más dificultades de relación social. Del mismo modo, se elaboró un ludograma a partir de la observación de la interacción en el juego de los 10 pases, para poder verificar la interacción del alumnado durante una clase de EF.

Todo ello encaminado a establecer el punto inicial sobre el que diseñar y preparar la fase activa. Como se indica en el apartado anterior, en esta fase se implementó la práctica la hibridación de modelos AC y EA. Con la intención de registrar la evolución de la experiencia práctica y poder analizarla a posteriori, se llevó un registro anecdótico. Para sistematizar el instrumento se diseño una tabla en la que se incluía la fecha de la sesión, el escenario didáctico, la recopilación de situaciones durante la práctica educativa y las reflexiones post-sesión e interpretación por parte del autor principal. Para el registro diario se realizaron anotaciones a modo de borrador, que posteriormente fueron revisadas y seleccionadas las más significativas y relevantes de cada sesión.
Ya en la fase postactiva, para poder conocer la percepción y valoraciones de los participantes en la experiencia se añadieron dos nuevos instrumentos. Para el alumnado, se diseñó un formulario de Google Forms ad hoc, ofreciendo un plazo al alumnado para su cumplimentación. En él se incluyeron preguntas de escala Likert dirigidas a conocer el grado de satisfacción del alumnado con la experiencia. Después se añadieron preguntas de tipo abierto para que el alumnado valorara los aspectos positivos y mejorables de la experiencia, así como preguntas relacionadas con el trabajo sobre las emociones realizado en las sesiones de tutoría. Finalmente, se propuso un espacio libre por si el alumnado quería añadir alguna información no contemplada en el instrumento.

$\mathrm{Al}$ docente se le realizó una entrevista en profundidad de carácter semiestructurado, una vez finalizada la intervención, cuya intención fue conocer su percepción acerca de la intervención realizada tras haber presenciado el desarrollo de la UD. Dicha entrevista fue grabada y posteriormente transcrita para su posterior análisis junto con la información obtenida en el registro anecdótico y el cuestionario de valoración del alumnado.

\section{Estrategias de investigación y análisis de datos}

Las estrategias de investigación seguidas en este proyecto son de carácter cualitativo. Concretamente, se optó por un diseño estudio de caso (el grupo-clase), pues, según Stake (2007), el caso contiene un elemento contextual que no solo lo dota de interés por sí mismo, sino por la capacidad de transferibilidad que puede tener hacia otros contextos semejantes. Además, el propio carácter contextual del trabajo de investigación de índole cualitativo hizo que en algún momento las propias estrategias de análisis de datos se reformularan, pues a medida que avanzaba la investigación desde la práctica, se reflexionaba acerca del diseño de la investi- 
gación y los posibles resultados que se obtendrían. Así pues, el proceso que se siguió para comparar, contrastar y analizar ha partido de la técnica de triangulación de fuentes que a su vez se estableció como criterio de veracidad (Cabrera, 2005). Para ello, se realizó la transcripción y posterior lectura de los datos recogidos en cada instrumento, como primera aproximación al análisis de los datos.

A continuación, se procedió a ir segmentando la información en diferentes unidades de significado que se fueron simplificando y resumiendo con la intención de obtener una cantidad de información accesible para la interpretación (tabla 2).

Posteriormente, las unidades de significado se fueron agrupando mediante un código de colores que permitía realizar una primera categorización visual. Seguidamente se fueron identificando puntos de unión que fueron dotándose de sentido, siguiendo los planteamientos de Strauss y Corbin (2002), hasta llegar a una propuesta de categorías de análisis. Estas fueron contrastadas, tanto con el docente de EF del centro como con el tutor que supervisaba las distintas fases del proceso de resultados. Finalmente, se realizó un proceso de inducción analítica tomando como referencia literatura especializada (Martínez, 2006; Rodríguez, Gil \& García, 1996) para agrupar la información en las categorías esenciales que se observan en la figura 1.

Tabla 2 .

Unidades de significado contenidas de las que derivan las categorías de análisis.

Unidades de significado contenidas de las que derivan las categorías de análisis.
\begin{tabular}{|l|l|l|}
\hline Unidades de significado & Categoría & Subcategoría \\
\hline $\begin{array}{l}\text { Problemas, interacciones, nivel de excitación, ayuda, solucionar, } \\
\text { hablado, diálogo, agresión, conflictos, mediador, valores. }\end{array}$ & Convivencia & $\begin{array}{l}\text { Resolución } \\
\text { de conflictos }\end{array}$ \\
$\begin{array}{l}\text { Sentimientos, daño, golpe, beneficios, autoestima, emociones, } \\
\text { conocernos, entenderme, controlarme, dejarse llevar, enfadados, } \\
\text { relajarme, actúa. }\end{array}$ & $\begin{array}{l}\text { Educación } \\
\text { emocional }\end{array}$ \\
\hline $\begin{array}{l}\text { Consenso, significativa, bienestar, interdependencias positivas, } \\
\text { colaborativa, trabajar, ayuda, amigo, implicado, gestos de ayuda, } \\
\text { dependían, competitividad, individualidad. }\end{array}$ & $\begin{array}{l}\text { Hibridación de } \\
\text { modelos }\end{array}$ & $\begin{array}{l}\text { Aprendizaje } \\
\text { cooperativo }\end{array}$ \\
\cline { 1 - 1 } $\begin{array}{l}\text { Retos, motivación, fructífero, emoción, interesante, animaba, } \\
\text { ayudaba, ganas, tensión, nervioso, expectación, interés, aventura }\end{array}$ & $\begin{array}{l}\text { Educación } \\
\text { aventura }\end{array}$ \\
\hline $\begin{array}{l}\text { Fantástica, adaptarnos, interesante, clima de clase, fundamentada, } \\
\text { mejora, entendido, aprendido, espectacular, positivo, avanzar, } \\
\text { calidad, mejor, exito, desarrollo, motivado, implicado, ayuda, } \\
\text { autonomía, actitud. }\end{array}$ & Evidencias de progreso \\
\hline
\end{tabular}
autonomía, actitud.

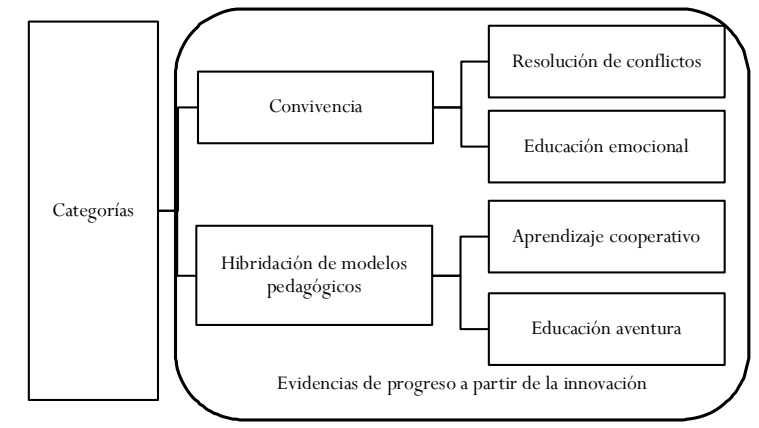

Figura 1. Mapa conceptual de la relación de categorías

\section{Resultados y discusión}

Experiencia de diseño, desarrollo y evaluación de una Unidad Didáctica (UD) en la que se hibridaron los modelos pedagógicos de EA y AC como estrategia metodológica para mejorar la convivencia y la gestión de conflictos desde las asignaturas de EF y la tutoría. El segundo es conocer las percepciones, valoraciones y el grado de satisfacción del alumnado y profesorado tras haber vivenciado la UD diseñada.

Tras haber descrito las fases del diseño y el desarrollo de la UD hibridada de los modelos pedagógicos de AE y AC y de haber indagado en las percepciones y visiones de los agentes participantes en la experiencia, en este apartado se trata de evidenciar y justificar en qué medida la intervención ha contribuido a la mejora de la convivencia entre iguales y la gestión de conflictos en la clase de EF. Los resultados se presentan diferenciando entre los obtenidos en la fase preactiva, de carácter más descriptivo y, los organizados por instrumentos y los obtenidos de la fase postactiva, mediante el método comparativo de datos de la triangulación de fuentes.

Del cuestionario dirigido al alumnado se constata su malestar en la escuela por los comportamientos de los compañeros, siendo que el $62 \%$ del alumnado a veces se siente mal y el $25 \%$ se siente mal cada día. Además, se manifiesta la percepción del alumnado que un $67 \%$ tiene conflictos cada día y un $25 \%$ a veces. Estos datos están en sintonía con lo expuesto por Abramovay (2005) sobre que el sistema educativo es afectado directamente por problemas de conflictividad y violencia deteriorando las relaciones sociales.

Otro dato evidente que se presenta en el mismo instrumento es la percepción del alumnado ante la reacción al conflicto, pues un 25\% huye del conflicto, no haciendo caso e ignorando a la persona que le ha molestado. Por lo tanto, una de las necesidades de trabajo era la de ofrecer al alumnado oportunidades de choques de intereses para que se creen oportunidades de discusión y que se deban resolver en el momento, no huyendo del conflicto. No obstante, es importante reconocer que otro $25 \%(8 \%+17 \%)$ contesta enfadado y suele decir palabrotas e insultos, incluso suele llegar a pegar al otro. En ese sentido, Hernández-Álvarez (2008) indica que la aparición de conflictos en la práctica educativa es una realidad manifiesta, pero puede suponer una oportunidad para afrontar positivamente las disputas. Asimismo, el alumnado considera que percibe en un 38\% (21\% el maestro castiga a los implicados $+17 \%$ el maestro habla con los implicados y busca una solución) que el docente es el que resuelve los conflictos. Esa figura del docente como mediador es reconocida por Moore 
(2014), en la que las partes acuden voluntariamente a una tercera persona neutral, nombrado mediador, ya que consideran que por sí solos no pueden llegar a solucionar sus problemas mediante el diálogo. A esta estrategia de mediación cabría añadir, en línea con el planteamiento de Pérez de Guzmán, Amador Muñoz y Vargas Vergara (2011), la conveniencia de resolver los conflictos en el momento y no esperar a que se resuelvan por sí mismos.

Por otro lado, el $46 \%$ del alumnado afirma que hay más conflictos en EF que en el aula o el patio. Asimismo, el 38\% reconoce que faltarse al respeto es el comportamiento causante principal de EF y, en segundo lugar, se enfadan en la creación de grupos, razones que justifican la conveniencia de abordar esta acción desde la EF. Asimismo, se añaden como datos más significativos la gran presencia de la competitividad en un $50 \%$, siendo el principal motivo por el que no se puede trabajar en EF. Por estos motivos, autores como Fraile-Aranda et al. (2008) abogan la incorporación de dinámicas como la asamblea o la reflexión grupal, más habituales en espacios como la tutoría o en asignaturas de aula, en la clase de EF, al considerarlas como apropiadas para la mediación y la resolución de conflictos.

En cuanto a los resultados del análisis sociométrico, el estudio del ludograma determina que el alumnado número 4, 5 y 16 son los que mayor grado de sociabilidad tienen en la clase, obteniendo un total de 9, 9 y 7 «Muy Bien» respectivamente. Mientras que los alumnos número 5, 14 y 18 han obtenido 8, 8 y 9 «Muy Mal». Otro resultado que se desprende del análisis del ranking sociométrico, es que se establece la moda con un total de 129 puntos la categoría de Regular. Por lo que sirve de indicador inicial de la necesidad de intervención didáctica para mejorar el nivel de cohesión del grupo clase. Estos resultados se ven reflejados sensiblemente con el ludograma, pues quienes mayor interacción han tenido en el resultado de 6 jugadas es el alumnado número 4, 15, 17 y 23. Esa interacción también se ve reflejada en los menos participativos, siendo el 22 y el 18. Por lo tanto, ambas herramientas coinciden en las personas con mayor grado de sociabilidad y en parte, con las de menor grado. Estos resultados han permitido reconocer las relaciones sociales del alumnado, lo que permitió diseñar las estrategias metodológicas para incidir en ello desde la UD hibridada.

Seguidamente, mostraremos los efectos que observamos de la intervención. Cabe indicar que se manifiestan como categorías y se reflejan las relaciones en la figura 1. Asimismo, exponer que los códigos utilizados a continuación sobre los que se presentan evidencias responden a los códigos del alumnado como [Al], los del especialista del área como [EEA] y, el registro anecdótico sistemático como alumno en prácticas [RAS]. Mientras que el número que les precede corresponde con el registro de la aportación.

\section{Convivencia}

La convivencia es un aspecto que se ha mostrado presente a lo largo de toda la puesta en práctica de la innovación. No obstante, se han reconocido 2 categorías que aparecen reflejadas en varios instrumentos y que hacen referencia a la convivencia:

\section{Resolución de conflictos}

En esta categoría se recogen aspectos de mejora por parte de alumnado, como elemento primordial en la investigación de la innovación. Hay quien al preguntarle sobre si consideran que trabajar las emociones en tutoría es bueno para ellos, alude a la importancia de aportar soluciones de la vida real, mediante ejemplos «si porque hemos hablado de situaciones nuestras solucionándolas dando ejemplos» [Al 19]. Mientras que también está el alumno que reconoce que intenta utilizar el diálogo «hablar» antes de agredir a alguna persona (pegar), «a no pegarles es que a veces se me iba la mano, ahora hablo si puedo» [Al 50].

Por otro lado, hay un grupo de alumnos / as que aporta su perspectiva dejando entrever que creen que han mejorado la forma de solucionar los problemas. Hay quien reconoce que se ha dado cuenta que no gritar e intentar decir las cosas en otro tono de voz le puede ayudar a solucionar sus problemas. Estas técnicas coinciden sensiblemente con lo aportado por Figueras, Calvo y Capllonch (2014) contemplando que la interacción el diálogo entre iguales, favorece la gestión del conflicto y la convivencia del grupo. Estas son algunas de las aportaciones que expresan lo comentado, «he aprendido a ayudarme de los amigos a solucionar un problema» $[\mathrm{Al}$ 44], o «a no gritar porque grito en el fútbol y aquí me sale solo intento hablar más tranquilo» $[\mathrm{Al} \mathrm{45}]$ y «muchas, pero la que más uso el diálogo» [ $\mathrm{Al} 48]$. Por lo que concierne al especialista, reconoce la realidad que dispone el alumnado de no saber solucionar sus propios problemas, «no obstante, hay situaciones en las que el alumnado se pelea puntualmente, pero la mayoría de las ocasiones los problemas vienen porque no saben solucionarlos.» [EEA].

Por otro lado, en el registro anecdótico se han recogido actuaciones de la práctica realizada que evidencia- 
ban situaciones en las que se requería de habilidades de resolución de conflictos y se han resuelto satisfactoriamente, «se ha mostrado una agresión verbal entre dos miembros del grupo por la competición. Sin intervenir como docente, ellos solos lo han resuelto porque querían seguir la práctica y el uno sin el otro no podían.» [RAS6].

\section{Educación Emocional}

De los resultados emerge la importancia de la educción emocional como elemento clave en la convivencia y las relaciones entre iguales. En la entrevista con el especialista, se dota de importancia a la EF, en el sentido que se dan más situaciones de agresiones físicas. «Se manifiestan más en actitudes físicas, lo cual no quiere decir que sean más importantes que otros más ocultos. Hay veces que se hieren los sentimientos y hace más daño que un golpe.» [EEA]. En esta cita se hace referencia a los sentimientos como elemento de mayor importancia a las «actitudes físicas». En consecuencia, se coincide con Sáez de Ocáriz, et al. (2014) en que los sentimientos y las emociones se conciben como factores fundamentales que influyen en la convivencia social, hasta el punto de consolidarse como base de posibles situaciones conflictivas. Por lo tanto, se reconoce a los sentimientos o las emociones como elementos que aparecen en el área. Asimismo, se manifiesta la utilidad del trabajo realizado, siendo de gran transferencia a la vida diaria del alumnado. Esto se ve reflejado en la cita, «que esas emociones que se han trabajado las puedan utilizar en cualquier ocasión de su vida diaria.» [EEA].

Por otro lado, el alumnado hace referencia a la empatía que se ha trabajado en clase, en el sentido de saber cómo se siente la otra persona. Como, por ejemplo, «me he sentido bien después de haber reconocido mis emociones y saber porque los demás lo hacen» [Al 15] o, «si a entender como hablar a los enfadados» [Al 49]. Por lo tanto, comparándolo con lo expuesto por Delors (citado por Fuertes \& Gabarró, 2013, p. 21) se constata que la educación emocional como herramienta para la prevención ya que los orígenes de muchos problemas están en el ámbito emocional.

Por otra parte, el registro anecdótico aporta actuaciones reconocidas por el alumnado en las que se reflejan aspectos comentados de educación emocional en los otros instrumentos. Por ejemplo, en el desarrollo de la empatía se recoge «en el debate final se sacan conclusiones interesantes como la de una alumna «es importante saber cómo se siente el personaje de las fotos para entender cómo actúa». [RAS1]. Así pues, se considera que mediante la formación en educación emocional que se ha llevado a cabo se ha contribuido a la mejora del comportamiento del alumnado. Esto sintoniza con lo desarrollado por Fuertes y Gabarró (2013) al hablar sobre beneficios en la educación emocional, pues no solamente puede contribuir en la mejora del comportamiento del alumnado, sino que son totalmente válidas para la mejora del rendimiento académico. Ello se puede entrever en las siguientes aportaciones ordenadas cronológicamente, «el dominó emocional ha dado mucho juego puesto que en la reflexión final el alunado comentaba situaciones que habían vivido y se daban las soluciones entre ellos mismos.» [RAS4]; «la actividad de «me gustaría y te felicito por» ha servido para aceptar las críticas de los compañeros. Este es un aspecto muy interesante puesto que sería de los últimos eslabones en el trabajo de la EE.» [RAS12].

\section{Hibridación de modelos pedagógicos}

El otro pilar fundamental en la investigación de la innovación es el reconocimiento de la mejora de la convivencia mediante la hibridación de los modelos pedagógicos utilizados. Para ello, se ha dividido el presente bloque en el AC y EA, analizando por separado la propuesta realizada sobre las características del modelo en cuestión.

\section{Aprendizaje cooperativo}

Uno de los aspectos más relevantes que muestran beneficios del trabajo del presente modelo es el expuesto por el especialista, «está más que demostrado la cantidad de beneficios aportados al alumnado en relación al establecimiento de roles, poner hipótesis, plantear soluciones, llegar a consenso, etc.» [EEA]. Estos beneficios aportados por el especialista, formarían parte de la característica de «habilidades sociales» concretada por Johnson, Johnson y Holubec (2013). Otro aspecto interesante destacado por el mismo es que en pocas ocasiones el AC da la oportunidad de mejorar el bienestar en el aula, aspecto que hace referencia a la convivencia entre iguales. Esto se percibe claramente cuando se le pregunta directamente si cree que el AC tiene relación directa con la convivencia, a lo que se responde «sí y con el bienestar dentro del aula, especialmente a aquellos alumnos excluidos socialmente. El $\mathrm{AC}$ es una oportunidad para incluirlos y que se creen interdependencias positivas.» [EEA]. Estas afirmaciones concuerdan con la teoría de Aubert, Bizkarra y Calvo (2014) sobre la que se determina que la EF es un elemento socializador que favorece la cohesión social. Por 
lo que concierne al alumnado, cabe reconocer la problemática que se representaba en el que se reconocían problemas en la creación de grupos. Estos aspectos, aparecen reconocido por un alumno, «me gusta todo menos cuando al principio me tocaba con quien no quería» [Al 7].

Sin embargo, se perciben aportaciones que hacen referencia que esa gestión del grupo ha sido superada. Esto se evidencia en el registro anecdótico, «la subdivisión de grupos con las pinzas era aleatoria y al principio han mostrado un carácter reticente a trabajar. Pero al ver que los demás grupos lo hacían igualmente, ha hecho que superaran esa barrera e intentar dar el doble de sí para compensar «ese grupo desigualado». [RAS8]

Por lo tanto, el AC ha aportado un beneficio real en el trabajo entre iguales. Las siguientes declaraciones denotan lo comentado, aportando beneficios del modelo pedagógico utilizado, tras cuestionarles qué creen que han aprendido de los «desafíos cooperativos»: «que con los demás se puede hacer mejor que yo solo» [Al 19], «que la gente ayuda más de lo que creía» $[\mathrm{Al} \mathrm{31]} \mathrm{y,} \mathrm{«que}$ da igual con el que trabaje si es amigo o no porque entre los dos podemos superarlos» [ $\mathrm{Al} 32]$.

También, desde el registro anecdótico se descubren mejoras del alumnado, puesto que se descubrieron gestos de ayuda, frases de motivación entre iguales e interdependencia positiva intragrupal. Esas interdependencias positivas encajan como una de las características esenciales del AC expuestas por Johnson, Johnson y Holubec (2013) siendo una dependencia entre las personas componentes del grupo para la consecución de los objetivos. Ello se puede percibir en las siguientes aportaciones: «se han implicado mucho más y al desplazarse por tríos mostraban gestos de ayuda, como por ejemplo que uno no podía correr bien (estaba recuperándose de una lesión) y los otros lo ayudaban cogiéndolo casi en el aire.» [RAS2]; «en el juego de metroagujero la individualidad ya no servía porque si todos no lo sujetaban no lo conseguían. Entonces se han visto frases de ayuda «vamos, cógelo, tú puedes» [RAS6]; «me ha sorprendido el juego de macarrones y espaguetis, ya que se ayudaban si uno solo no podía coger el macarrón. Uno se lo acomodaba para que la otra pudiera cogerlo. Se manifestaba la interdependencia positiva por primera vez.» [RAS7].

Finalmente, teniendo en cuenta el global del análisis de los datos extraídos, se puede determinar que en cierto modo el modelo de AC ha podido llegar a contribuir, tanto en la convivencia y las habilidades sociales, como en la motivación del alumnado, hecho que como indican (Bermejo Díaz, et al., 2021), mejoran los resultados del trabajo en la clase de EF. Esta afirmación se corresponde con los resultados encontrados en Sáez de Ocáriz, et al. (2014) sobre que el trabajo cooperativo puede llegar a optimizar el desarrollo de habilidades y competencias sociales ayudando a mejorar la convivencia escolar.

\section{Educación Aventura}

Por lo que refiere al modelo de EA y por parte del especialista, se destaca la motivación que aporta el modelo en cuestión, siendo aspecto que puede promover mejoras, «aventura y la motivación siempre van unidas por lo tanto plantear cualquier contenido en otras áreas de esta manera siempre va a ser fructífero.» [EEA]. Extrapolando esa motivación al área de EF. SánchezAlcaraz (2014) destaca el área de EF como una disciplina motivante para el alumnado y promotora de relaciones interpersonales y comunicación brinda la oportunidad de desarrollar cualidades sociales y personales como la autoestima, la solidaridad o la cooperación. Estos datos coinciden con la percepción del alumnado en las contribuciones recogidas en el formulario al preguntar sobre el aspecto motivacional de los desafíos. Algunos detallan la incertidumbre que vivían y el interés por los mismos en, «molaban mucho porque eran inquietantes» $[\mathrm{Al} \mathrm{34}]$ y «me hacían estar en tensión» [Al 38]. Esa incertidumbre registrada es considerada por Priest y Gass (2005) como un proceso de la EA en el que los participantes intervienen en actividades, tareas y juegos con un componente de incertidumbre y cierto riesgo subjetivo, entremezclándolo con propósitos de confianza, reto, compañerismo, etc.

También se han registrado aportaciones que hacen referencia al medio en el que se desarrollaba, por ejemplo «sí porque nunca se hacen fuera del gimnasio y eso mola» [Al 36]. Esta cita corresponde con la encontrada en el registro anecdótico, siendo «La aventura de adentrarse y perderse por el colegio hacía que su motivación aumentara considerablemente.» [RAS8]. Ese aumento de la motivación en el medio natural es destacado por Baena-Extremera (2011, p.4), pues «el medio natural es un lugar lleno de estímulos para desarrollar a los alumnos, sobre todo a nivel psicológico». De igual modo, cabe reconocer el factor de la motivación como elemento que la gran mayoría de docentes utilizan para para la promoción del éxito de la propia asignatura (Ortíz, Allepuz \& Sánchez, 2019). Mientras que el registro anecdótico refleja la tensión vivida por el alumnado, fruto del riesgo real que ofrecían la naturaleza de las tareas propuestas, «los desafíos los ponían en 
tensión y eso se manifestaba en frases como, con esta música de fondo y el tiempo me motivo mucho y me pongo nervioso». [RAS6].

\section{Evidencias de progreso a partir de la innova- ción}

La última categoría pone de manifiesto aspectos de progreso que aparecen reflejados en las demás categorías y que, aun estando relacionadas, no encajaban en una en concreto, por lo que se ha decidido aglutinarlas en este código.

En primer lugar, se han encontrado citas que apoyan la adecuada puesta en práctica de la propuesta didáctica. El especialista, reconoce el gran esfuerzo realizado desde la innovación por adaptarse al alumnado y la fundamentación metodológica. Esto se muestra en frases como «creo que la intervención fue fantástica porque el alumno se adaptó al ritmo de trabajo del IES con interés y esfuerzo. Colaboró en todo lo que se le propuso e intervino en la impartición de una unidad didáctica de trabajo cooperativo, desafíos y emociones muy bien fundamentada metodológicamente.» [EEA]. Asimismo, tras preguntar acerca de si considera que la intervención había servido para mejorar la convivencia del alumnado, el mismo alude «este tipo de intervención facilita el clima de clase y mejora la cohesión del grupo. Y eso es fundamental para otras tareas de aprendizaje cooperativas en cualquier asignatura. Además, el hecho de enfrentarlos mediante desafíos de incertidumbre les ha venido muy bien.» [EEA]. Mientras que el alumnado cuando se le pregunta acerca de la convivencia después de la intervención afirma que se llevan «mejor ya que solucioné lo mío con otro que me llevaba mal. Ahora bien ya» [Al 53], «mejor porque al jugar juntos molaba i nos hablábamos» [Al 57] y, «mejor me gusta arreglar las cosas me siento mejor luego» [Al 55]. Por tanto, tanto profesorado como alumnado coincide en que la afrontación del conflicto mediante estrategias de trabajo cooperativo les ha venido bien, en tanto que les ha permitido aprender a que el diálogo y la colaboración son una alternativa que, a diferencia de la discusión y la confrontación, les permite solucionar sus diferencias.

Otro resultado evidente es el aportado por el especialista, «en este sentido, has sabido llevar a cabo las características principales de este método y el alumnado ha aprendido específicamente esa forma de trabajo.» [EEA]. Mientras que, al hibridarlo con la EA, añade que se había conseguido sacar el aspecto positivo de ambos modelos, «creo que la aventura lo afecta de por sí desde la competitividad, por lo tanto, se promocionan aspec- tos negativos y que van en contra del AC. Pero si se juntan el resultado es espectacular, la UD que has hecho era eso y sacaba el aspecto positivo de los dos modelos.» [EEA]. Estas apreciaciones concuerdan con la calificación de la innovación realizada por el alumnado que valora con una media de 7,7 la experiencia y una moda de 9, los aprendizajes que considera haber adquirido. Estos resultados están en consonancia con experiencias previas de hibridación de los modelos pedagógicos de AC y EA. Concretamente, Fernández-Río (2015), obtuvo que la cooperación fue un elemento esencial para garantizar la seguridad en las tareas de EA. Asimismo, el propio Fernández-Río y Méndez-Gimenez (2016) afirman que la hibridación de modelos contribuye a optimizar los objetivos de aprendizaje y desarrollo personal del alumnado, hecho que refuerza la conclusión del estudio de Peiró-Velert y Méndez-Giménez (2017), que consideran que un correcto planteamiento de modelos pedagógicos hibridados amplía los beneficios que se obtendrían planteando de los modelos por separado.

\section{Conclusiones}

El presente estudio muestra el papel que juega la hibridación de los modelos pedagógicos de aprendizaje cooperativo y educación aventura, junto al trabajo en tutoría, en la educación emocional del alumnado. Los resultados obtenidos evidencian una contribución positiva en la gestión de conflictos y la convivencia entre iguales de un alumnado que previamente mostraba carencias en la convivencia y la resolución de conflictos. Además, se ha observado cómo el trabajo cooperativo y los contenidos de aventura han mejorado la motivación y el interés del alumnado por la actividad en la clase de EF. Por tanto, se podría concluir que la hibridación de los modelos pedagógicos se ha mostrado como una buena contribución a la didáctica de la EF, en la medida en la que permite la integración de contenidos transversales y propios de la asignatura de Educación Física, que revierten en nuevas posibilidades de aprendizaje al alumnado.

\section{Referencias}

Abramovay, M. (2005). Victimización en las escuelas. Ambiente escolar, robos y agresiones físicas. Revista mexicana de investigación educativa, 10(26), 833-864.

Aubert, A., Bizkarra, M. \& Calvo, J. (2014). Actuaciones educativas de éxito desde la Educación Física. Retos. Nuevas tendencias en Educación Física, Deporte y 
Recreación, 25, 144-148.

Baena-Extremera,A. (2011). Programas didacticos para educacion fisica a traves de la educacion de aventura. Espiral. Cuadernos del Profesorado, 4(7), 3-13.

Bermejo Díaz, J. M., Pulido Salas, D., Galmes-Panades, A. M., Serra Payeras, P., Vidal-Conti, J., \& Ponseti Verdaguer, F. J. (2021). Educación física y universidad: Evaluación de una experiencia docente a través del aprendizaje cooperativo. Retos. Nuevas tendencias en Educación Física, Deporte y Recreación, 2021, 39, 90 97.

Caballero, P., Sayazo, D., Dominguez, G., Perez, O. \& Parra, M. (2006). Jugando en un espacio de fantasia. Claves para elaborar una jornada de aventura en un centro de ensenanza. Retos. Nuevas tendencias en Educacion Fisica, Deporte y Recreacion, 10, 23- 30.

Cabrera, F.C. (2005). Categorización y triangulación como procesos de validación del conocimiento en investigación cualitativa. theoria, 14(1), 61-71.

Fernández-Río, J. (2015). El Modelo de Responsabilidad Personal y Social y el Aprendizaje Cooperativo. Conectando Modelos Pedagógicos en la teoría y en la práctica de la Educación Física. In Actas del IV Congreso Internacional de Educación Física. Querétaro, México.

Fernández-Rio, J., Calderon, A., Hortiguela, D., PerezPueyo, A. \& Aznar-Cebamanos, M. (2016). Modelos pedagogicos en educacion fisica: consideraciones teorico-practicas para docentes. Revista Espanola de Educacion Fisica y Deportes, 413, 55-75.

Fernández-Rio, J. \& Mendez-Gimenez, A. (2016). El aprendizaje cooperativo: modelo pedagogico para educacion fisica. Retos. Nuevas Tendencias en Educacion Fisica, Deporte y Recreacion, 29, 201- 206.

Figueras, S., Calvo, J., \& Capllonch, M. (2014). Prevención y resolución de conflicto en Educación Física desde la perspectiva de los adultos miembros de la comunidad educativa en las comunidades de aprendizaje. Retos. Nuevas tendencias en Educación Física, Deporte y Recreación, 25, 168-173.

Fraile-Aranda, A., López-Pastor, V.M., Ruíz-Omeñaca, J.V., \& Velázquez-Callado, C. (2008). La resolución de los conflictos en ya través de la educación física. 251, Barcelona: Graó.

Fuertes, I., \& Gabarro, M. (2013). Una altra mirada a les tutories $\left(1^{\mathrm{a}} \mathrm{ed}.\right)$. Barcelona: Associacio de Mestres Rosa Sensat.

Funes, S. (2000). Resolución de conflictos en la escuela: una herramienta para la cultura de paz y la convivencia. Contextos educativos: Revista de Educación 3, 91 -
106.

García-Hierro, M. A., \& Cubo, S. (2009). Convivencia escolar en Secundaria: aplicación de un modelo de mejora del clima social. Revista Electrónica Interuniversitaria de Formación del Profesorado, 12(1), 51-62.

González-Víllora, S., Evangelio, C., Sierra-Díaz, J., \& Fernández-Río, J. (2019). Hybridizing pedagogical models: A systematic review. European Physical Education Review, 25(4), 1056-1074. Doi: https:// doi.org/10.1177/1356336X18797363

González, V. M., Gómez-López, M., \& Granero-Gallegos, A. (2019). Relación entre la satisfacción con las clases de educación física, su importancia y utilidad y la intención de práctica del alumnado de Educación Secundaria Obligatoria. Revista Complutense de Educación, 30(2), 479. Doi: https: / /doi.org/10.5209/ RCED. 57678

Guijarro, E. Evangelio, C. González-Víllora, S \& AriasPalencia, N. M. (2020). Hybridizing Teaching Games for Understanding and Cooperative Learning: an educational innovation. ESHPA - Education, Sport, Health and Physical Activity. 4(1): 49-62.

Hernández-Alvarez, J.L. (2008). Educacion Fisica, deporte y educacion en valores. Conocimiento, Educacion yValores, 8, 1-16.

Hortiguela, D., Perez-Pueyo, A. \& Fernández-Rio, J. (2020). Evaluacion formativa y modelos pedagogicos: modelo de responsabilidad personal y social y de autoconstruccion de materiales. Revista Espanola de Educacion Fisica y Deportes, 430, 23-41.

Johnson, D. W. \& Johnson, R. T. (1999). Aprender juntos y solos. Aprendizaje cooperativo, competitivo e individualista. Buenos Aires: Aique.

Johnson, D. W., Johnson, R., \& Holubec, E. (2013). Cooperation in the classroom ( $9^{\mathrm{a}}$ ed.). Edina, MN: Interaction Book Company.

Jiménez-Barbero, J. A., Jiménez-Loaisa, A., GonzálezCutre, D., Beltrán-Carrillo, V. J., Llor-Zaragoza, L., \& Ruiz-Hernández, J. A. (2020). Physical education and school bullying: a systematic review. Physical Education and Sport Pedagogy, 25(1), 79-100. Doi: https: / / doi.org/10.1080/17408989.2019.1688775

Lamoneda Prieto, J., González-Víllora, S., \& FernándezRío, J. (2020). Hibridando el Aprendizaje Cooperativo, la Educación Aventura y la Gamificación a través de la carrera de orientación. Retos. Nuevas tendencias en Educación Física, Deporte y Recreación, 38(38), 754-760.

Lizandra, J. \& Peiró-Velert, C. (2020). Las relaciones 
sociales y su papel en la motivación hacia la práctica de actividad física en adolescentes: Un enfoque cualitativo. Retos: nuevas tendencias en educación física, deporte y recreación, 37, 41-47.

Martín, M., \& Ríos, O. (2014). Prevención y resolución del conflicto en educación física desde la perspectiva del alumnado. Retos. Nuevas tendencias en Educación Física, Deporte y Recreación, 25, 162-167.

Martínez, M. (2006). La investigación cualitativa, Revista IIPSI, 9(1), 123-146. Doi: http://dx.doi.org/ 10.15381/rinvp.v9i1.4033

Metzler, M.W. (2017). Instructional models for physical education ( $3^{\mathrm{a}}$ ed.). New York: Taylor \& Francis.

Monzonís, N. (2015). La Educación Física como elemento de mejora de la Competencia Social y Ciudadana. Investigación-Acción en torno a la aplicación de un programa basado en la prevención y resolución de conflictos desde el área Educación Física. Tesis doctoral. Universidad de Barcelona.

Moore, C. (2014). The Mediation Process: Practical Strategies for Resolving Conflict. San Francisco: Jossey-Bass.

Moreno, A. B. M. (2010). La acción tutorial en educación. Hekademos. Revista educativa digital, 7, 95-114.

Navarro, D., Collado, J. A. \& Pellicer, I. (2020). Modelos pedagógicos en Educación Física.

Ortíz, D. C., Allepuz, J. P., \& Sánchez, M. L. Z. (2019). Estado actual de la educación física desde el punto de vista del profesorado. Propuestas de mejora. Retos. Nuevas tendencias en Educación Física, Deporte y Recreación, 35, 47-53.

Palacio, E. S., \& Bermejo-García, S. (2021). Efectividad del desarrollo del autocontrol a través de la Educación Física. Revista Iberoamericana de Ciencias de la Actividad Física y el Deporte, 10(2), 126-139. Doi: https://doi.org/ $10.24310 /$ riccafd.2021.v10i2.11160

Parra, M., Caballero, P. \& Dominguez, G. (2009). Pedagogía de la Aventura. In Ma E., García Montes (coord.), Dinamicas y estrategias de recreación, 199-260, Barcelona: Graó.

Peiró, C. \& Julián, J. A. (2015). Los modelos pedagógicos en educación física: un enfoque más allá de los contenidos curriculares. Tándem:Didáctica de la educación física, 50(4), 9-15.

Peiró-Velert, C. P., \& Méndez-Giménez, A. M. (2017). Modelos pedagógicos en educación física. Tándem: Didáctica de la educación física, (57), 4-6.

Pérez de Guzmán, M.V., Amador Muñoz, L.V., \& Vargas Vergara, M. (2011). Resolución de conflictos en las aulas: Un análisis desde la investigación-acción. Pedagogía Social Revista Interuniversitaria, 8, 99-114. Doi: 10.7179/PSRI_2011.18.08

Pérez-Pueyo, Á., Alcalá, D. H., Álvarez, I. H., Cobo, D. V., Bernardino, C. H., García, J. G., ... \& Garijo, A. H. (2017). La hibridación de modelos pedagógicos en educación física y la evaluación formativa. Revista Infancia, Educación y Aprendizaje, 3(2), 411-418. Doi: https://doi.org/10.22370/ieya.2017.3.2.757 Priest, S. \& Gass, M. (2005). Effective leadership in adventure programming. Champaign, IL: Human Kinetics.

Rodríguez, G., Gil, J., \& García, E. (1996). Metodología de la Investigación Cualitativa. Málaga: Algibe.

Sáez de Ocáriz, U., Lavega, P., Mateu, M. \& Rovira, G. (2014). Emociones positivas y educación de la convivencia escolar. Contribución de la expresión motriz cooperativa. Revista de Investigación Educativa, 32(2), 309-326. Doi: https://doi.org/10.6018/ rie.32.2.183911

Sánchez-Alcaraz, B. J. (2014). La actividad fisica y el deporte como medio para el desarrollo personal y social en jóvenes escolares. Tesis doctoral. Universidad de Murcia.

Stake, R. (2007). Investigación con estudio de casos. Madrid: Morata.

Strauss, A., \& Corbin, J. (2002). Bases de la investigación cualitativa:Técnicas y procedimientos para desarrollar la teoría fundamentada. Medellín: Universidad de Antioquia.

Valero-Valenzuela, A., Gregorio Garcia, D., Camerino, O., \& Manzano, D. (2020). Hybridisation of the Teaching Personal and Social Responsibility Model and Gamification in Physical Education. Apunts. Educacion Fisica y Deportes, 141, 63-74. Doi: https:// doi.org/10.5672/apunts.2014-0983.es.(2020/ 3). 141.08

Velazquez, C. (2012). El aprendizaje cooperativo en educacion fisica. La formacion de los grupos y su influencia en los resultados. Tandem, 39, 75-84.

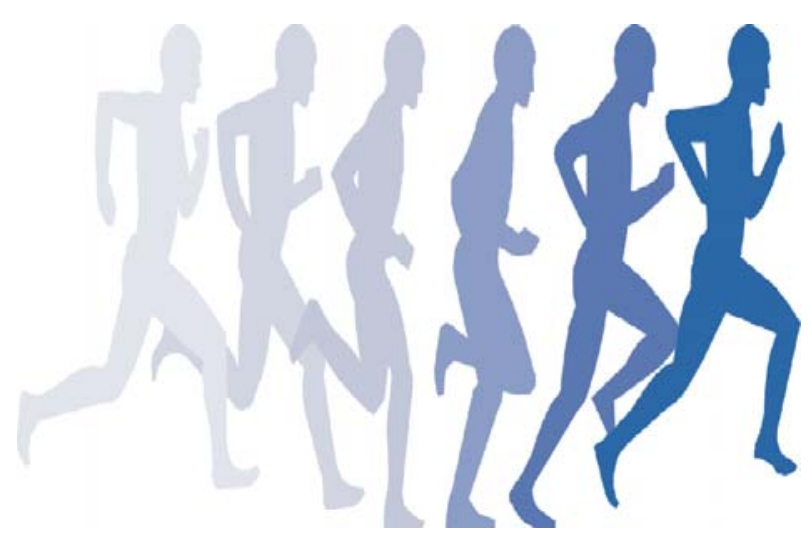

